\title{
GREEN WALKS IN A HYPERGRAPH
}

BY

\section{WOLFGANG RUMP (EICHSTÄTT)}

Introduction. For a finite group $G$, the blocks $\Lambda$ of $\widehat{\mathbb{Z}}_{p} G$ with cyclic defect are described by their Brauer tree. This tree has to be understood in conjunction with an embedding into the plane, and a clockwise "walk around the tree" [2] corresponds to a cyclic projective resolution in which each indecomposable projective $\Lambda$-lattice occurs exactly twice $[2,4,7]$.

In a recent paper, Roggenkamp [8] introduced a class of orders $\Lambda$ over a complete noetherian local domain $R$ which are similarly associated with a combinatorial object $G$ like a Brauer tree, such that there are projective resolutions of $\Lambda$-modules corresponding to Green walks in $G$. Namely, $G$ may be an arbitrary finite connected graph, possibly with "truncated" edges, that is, a kind of generalized edges which are attached to one vertex only. In contrast to a tree, a graph $G$ (with or without truncated edges) does not always admit an embedding into the plane. Therefore, one has to specify a local embedding in this case, or equivalently, a cyclic ordering of the germs of edges at every vertex. Let us refer to this concept as a TC-graph $(\mathrm{T}=$ truncated, $\mathrm{C}=$ cyclic ordering).

In the following example, there are two truncated edges $f$ and $g$.

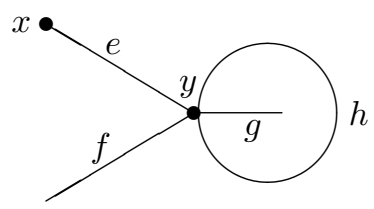

If the cyclic ordering at vertex $y$ is given by the clockwise orientation of the plane, there are two finite Green walks $f \rightarrow e \rightarrow e \rightarrow h \rightarrow f$ and $g \rightarrow h \rightarrow g$ which correspond to a pair of finite projective resolutions for the associated $R$-order (cf. [8]; also Example 2 of $\S 4$ below). In fact, Roggenkamp [8] shows that the occurrence of finite Green walks depends on the presence of truncated edges, whereas for a graph $G$ without truncated edges, there are only cyclic Green walks in $G$.

1991 Mathematics Subject Classification: Primary 16G30; Secondary 20C10, $05 \mathrm{C} 65$. 
In the present note, we generalize TC-graphs by a natural concept, quite easy to define, which occurs as underlying structure of a wide class of rings $\Lambda$ (including the orders considered in [8]), with projective resolutions according to Green's walk. Namely, we define a (finite) cycle hypergraph $H$ simply by a map $\varepsilon: C \rightarrow E$ between finite sets, together with a permutation $\pi$ on $C$. Then the cycles of $\pi$ are the vertices, the elements of $E$ the edges, and $\varepsilon$ gives the rule of attachment between vertices and edges.

For example, consider the set $C=\{1,2,3,4,5,6\}$ with permutation $\pi=$ (1) (23456), and define a cycle hypergraph $H$ by $\varepsilon: C \rightarrow E=\{e, f, g, h\}$ with $\varepsilon(1)=\varepsilon(5)=e, \varepsilon(2)=g, \varepsilon(3)=\varepsilon(6)=h, \varepsilon(4)=f$. Then there are two vertices $x=\{1\}, y=\{2,3,4,5,6\}$, and $H$ coincides with the TC-graph depicted above.

In general, any edge $e \in E$ may be attached to arbitrarily many vertices, possibly with multiplicities. For instance, a graphical loop, such as $h$ in the preceding TC-graph, has to be regarded as an edge with a twofold attachment to its vertex. Apart from these multiplicities of attachment, our notion of hypergraph coincides with that of Berge [1]. Being more general, however, our concept allows us to distinguish between 1-loops, i.e. edges with one vertex of multiplicity one, and 2-loops, i.e. edges with one vertex of multiplicity two as in a usual graph. (In fact, the 1-loops or "truncated edges" [8] are just the loops that occur in matroid theory [1, 10].) Thus if a cycle hypergraph has edges with at most two vertices (multiplicities counted) then it is equivalently given by a TC-graph.

Next let us associate an $R$-order $\Lambda_{H}$ with $H$. Assume first that $R$ is onedimensional, i.e. a complete discrete valuation domain. Then for the given permutation $\pi$ on $C$ there exists a basic hereditary order $\Gamma$ with an indecomposable $\Gamma$-lattice $P_{c}$ for each $c \in C$ such that $\operatorname{Rad} P_{c} \cong P_{\pi c}$. Secondly, the partition on $C$ induced by $\varepsilon$ gives rise to a subalgebra of $\Gamma / \operatorname{Rad} \Gamma$ which corresponds to a suborder $\Lambda_{H}$ of $\Gamma$ with $\operatorname{Rad} \Lambda_{H}=\operatorname{Rad} \Gamma$. If we require that $\Lambda_{H}$ is totally split, i.e. $\Lambda_{H} / \operatorname{Rad} \Lambda_{H}$ is a product of fields $\mathfrak{k}:=R / \operatorname{Rad} R$, then up to isomorphism, $\Lambda_{H}$ is uniquely determined by $H$. A characterization of such totally split orders $\Lambda_{H}$ as a special class of Bäckström orders will be given in Theorem 1.

Before we consider more general rings related to $H$, let us say a word about Green walks and projective resolutions in the case of an arbitrary finite cycle hypergraph $H$. We shall associate with $H$ a directed graph $W_{H}$ with vertex set $C$ such that for each $c \in C$, the arrows in $W_{H}$ starting from $c$ point to the $r$ possible continuations $c_{1}, \ldots, c_{r} \in C$ of a Green walk. (In contrast to classical Green walks, there may be several continuations!) In terms of projective resolutions, this means that the syzygy of the $\Lambda_{H}$-module $P_{c}$ is $P_{c_{1}} \oplus \ldots \oplus P_{c_{r}}$. From this it follows that the syzygies of the simple $\Lambda_{H}$-modules are given by $W_{H}$. Moreover, there is a one-to-one correspon- 
dence $e \mapsto P_{e}$ between the edges $e$ of $H$ and the indecomposable projective $\Lambda_{H}$-modules $P_{e}$, and the projective cover of the $\Lambda$-module $P_{c}$ is just $P_{\varepsilon(c)}$. Therefore, a projective resolution of $\Lambda_{H} / \operatorname{Rad} \Lambda_{H}$ can be described entirely in terms of $H$.

For a TC-graph $G$, our $R$-order $\Lambda_{G}$ coincides with Roggenkamp's order $\Lambda(G)([8]$, Definition 4.5) in the totally split case. More generally, it is shown in [8] that the hereditary order $\Gamma$ corresponding to our permutation space $C$ can be replaced by an arbitrary QH-order [7], that is, an $R$-order $\Gamma$ with a regular element $\omega \in \operatorname{Rad} \Gamma$ such that $\Gamma / \Gamma \omega$ is a product of local algebras. Then the relationship between Green walks and projective resolutions remains valid without change. Moreover, the dimension of $R$ does not affect this correspondence.

Now let us return to an arbitrary finite cycle hypergraph $H$. In $\S 4$ we associate with $H$ a most general class of rings $\Lambda$ for which the correspondence between Green walks and projective resolutions is valid. For any semiperfect $\operatorname{ring} S$, we define a (two-sided) ideal Pro $S \subset \operatorname{Rad} S$ and call it the proradical of $S$. We call $S$ prohereditary if there is an invertible ideal ( $\S 3$ ) $J$ between Pro $S$ and Rad $S$ with $\bigcap J^{i}=0$. (If $S$ has no local ring-direct factors, then $J$ necessarily coincides with Pro $S$.) We then prove that every QH-order is prohereditary, and that for every prohereditary $\operatorname{ring} \Gamma$ with defining ideal $J$ as above, the correspondence between Green walks and projective resolutions holds for a class of subrings $\Lambda$ of $\Gamma$ with Pro $\Lambda \subset J \subset \Lambda$.

We owe thanks to the referee for the suggestion to present our results in a general form (instead of restricting our exposition to totally split orders), so that the known examples of Brauer tree orders (e.g. blocks with cyclic defect) are explicitly covered by the article.

1. Hypergraphs. Let us define a hypergraph $H=(X ; E)$ as a function

$$
E \times X \rightarrow \mathbb{N}
$$

associating with each pair $(e, x)$ a non-negative integer $e x$, such that for each $e \in E$, and each $x \in X$,

$$
\mathrm{r}(e):=\sum_{y \in X} e y>0, \quad \mathrm{r}(x):=\sum_{f \in E} f x>0 .
$$

The elements of $X$ (resp. $E$ ) are called vertices (resp. edges), and $H$ is said to be finite if $X$ and $E$ are finite sets. The expressions (2) will be called the rank of $e$ and $x$, respectively; they can be infinite if $H$ is. The supremum $\sup _{e \in E} \mathrm{r}(e)$ (resp. $\sup _{x \in X} \mathrm{r}(x)$ ) defines the rank (resp. corank) of the hypergraph $H$. If $\mathrm{r}(e)=2$ for all $e \in E$, then $H$ is said to be a graph. In fact, there are two kinds of edges in this case: edges $e \in E$ which connect two different vertices $x \neq y$, i.e. $e x=e y=1$, and loops $e$ with $e x=2$ for 
a single $x \in X$. For an arbitrary hypergraph $H$, the function (1) gives the multiplicity of attachment between vertices and edges.

Our terminology generalizes that of C. Berge [1] whose (finite) hypergraphs arise if the function (1) is restricted to values 0 and 1 . Therefore, he is bound to define a loop (boucle) as an edge of rank one. Generalizing this, we define a loop as an edge $e$ with $e x \neq 0$ for just one vertex $x$. So there are loops of all different ranks $r>0$, briefly called $r$-loops. From a graph-theoretical point of view, the 1-loops could be imagined as "half" edges with one free end not attached to a vertex. They have been introduced by K. W. Roggenkamp [8] as truncated edges in order to generalize Green's "walk around the Brauer tree" [2]. Note that our definition of a hypergraph is dual with respect to vertices and edges.

In order to generalize the notion of a Brauer graph, let us define a permutation space as a set $C$ together with a bijection $\pi: C \rightarrow C$. For any $c \in C$, a successor $\pi c$ and a predecessor $\pi^{-1} c$ are uniquely defined. If $\pi$ operates transitively on $C \neq \emptyset$, we shall call $C$ a cycle. In this case, $\pi$ generates a cyclic group $\mathbb{Z} / n \mathbb{Z}$ of permutations on $C$, which determines $C$ up to isomorphism. Hence there is a one-to-one correspondence between non-negative integers $n$ and cycles $\mathbf{Z}_{n}$. Note that the infinite cycle is denoted by $\mathbf{Z}_{0}$. The following generalization of the well-known cycle decomposition of a (finite) permutation is obvious:

Proposition 1. Every permutation space $C$ has a unique decomposition into cycles.

Now let us define a cycle hypergraph $H$ as a permutation space $C$ together with a surjective map $\varepsilon: C \rightarrow E$ such that the set $X$ of cycles in $C$ satisfies

$$
e x:=\left|\varepsilon^{-1}(e) \cap x\right|<\infty
$$

for all $x \in X$ and $e \in E$. Then (3) defines a hypergraph with vertex set $X$ and edge set $E$ since

$$
\mathrm{r}(x)=|x|, \quad \mathrm{r}(e)=\left|\varepsilon^{-1}(e)\right| .
$$

If $H$ is finite and of rank $\leq 2$, this concept is equivalent to Roggenkamp's truncated graph with local embedding into the plane [8]. In fact, for each vertex $x \in X$, the elements $c \in x$ can be regarded as the germs of edges $\varepsilon(c)$ at $x$, and the cycle structure on $x$ is equivalent to a local plane embedding at $x$.

EXAMPLE 1. In the example given in the introduction, we have two edges $f, g$ of rank one, and two edges $e, h$ of rank two. The vertex $x$ is of rank one, whereas $y$ has rank five. Hence, this cycle hypergraph is of rank two and corank five. In principle, the TC-graph notation could be extended to cycle hypergraphs of rank $\geq 3$ if the edges of rank $\geq 3$ are depicted by means of 
star-shaped regions. However, with increasing corank, even for rank $\leq 2$, it will be more appropriate to draw the partition on $C$ induced by $\varepsilon$ together with the cycles in $C$. The above example then takes the following form:

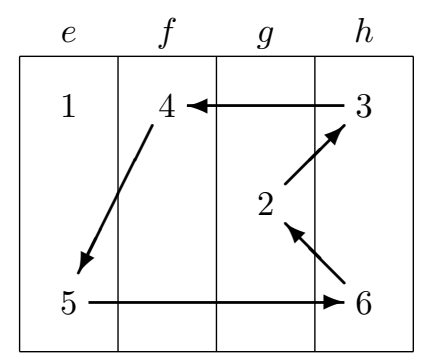

Examples of higher rank will be given at the end of $\S 4$.

Now let us define Green walks in an arbitrary cycle hypergraph $H$ given by a structure map $\varepsilon: C \rightarrow E$. For each $c \in C$, we introduce arrows $c \rightarrow \pi d$ for all $d \neq c$ with $\varepsilon(d)=\varepsilon(c)$. Then these arrows determine a directed graph $W_{H}$ with vertex set $C$ which we shall call the resolution graph of $H$. If $H$ is of rank $\leq 2$, then the directed paths in $W_{H}$ are just the Green walks defined in [8]. Therefore, we define a Green walk in $H$ as any directed path in $W_{H}$, starting at some $c \in C$ and continued as far as possible. Thus for higher rank of $H$, there may be several continuations at each vertex of such a walk. (This generalizes the fact that in a TC-graph, the number of possible continuations is zero or one.) In $\S 4$ we shall see that $W_{H}$ embodies the combinatorial structure of projective resolutions of certain modules over the rings associated with $H$.

2. The order of a cycle hypergraph. Let $R$ be a fixed complete discrete valuation domain with quotient field $K$, valuation ideal $\mathfrak{p}=\operatorname{Rad} R$, and residue field $\mathfrak{k}$. For the theory of $R$-orders and their representations we refer to [5].

In this section we shall start with any finite cycle hypergraph $H$ and associate with $H$ a canonical $R$-order $\Lambda_{H}$. These orders $\Lambda_{H}$ will be the prototype of orders for which a correspondence between Green walks and projective resolutions holds. Their internal characterization as a special class of Bäckström orders (Theorem 1) will then suggest the definition of a most general class of rings $\Lambda$ with an underlying cycle hypergraph $H$ for which such a correspondence is valid. These rings $\Lambda$ independent of the ground ring $R$ will be introduced in $\S 4$.

In the present section we shall consider $R$-orders $\Lambda$ (in a semisimple $K$ algebra) with the property that each simple $\Lambda$-module is of $\mathfrak{k}$-dimension one. Let us call them totally split orders. Equivalently, this says that $\Lambda / \operatorname{Rad} \Lambda$ 
is a $\mathfrak{k}^{- \text {-algebra }} \mathfrak{k}^{n}=\mathfrak{k} \times \ldots \times \mathfrak{k}$ for some $n \in \mathbb{N}$. Hence the set $\operatorname{Hom}_{R \text {-alg }}(\Lambda, \mathfrak{k})$ of $R$-algebra homomorphisms into $\mathfrak{k}$ has $n$ elements which can be identified with the simple $\Lambda$-modules.

For the following construction, note first that there is a contravariant equivalence between the category of $\mathfrak{k}$-algebras $\mathfrak{k}^{n}$ and the category set of finite sets, given by the functors $\operatorname{Hom} \mathfrak{k}$-alg $(-, \mathfrak{k})$ and $\operatorname{Map}(-, \mathfrak{k})$. If we replace $\mathfrak{k}$ by the quotient field $K$ of $R$, it follows that the functors $\operatorname{Hom}_{R \text {-alg }}(-, R)$ and $\operatorname{Map}(-, R)$ establish a duality between the category of $R$-orders $R^{n}$ in $K^{n}$ and set.

Construction of $\Lambda_{H}$. Let $H$ be a finite cycle hypergraph $H$ with structure map $\varepsilon: C \rightarrow E$. The permutation $\pi^{-1}$ on $C$ corresponds to an algebra automorphism $\tau$ of $\mathfrak{k}^{n}$, where $n=|C|$, and $\varepsilon$ corresponds to a $\mathfrak{k}^{-}$-subalgebra $B$ of $\mathfrak{k}^{n}$. For each cycle $\mathbf{Z}_{m}$ of $C$, there is a factor algebra $\mathfrak{k}^{m}$ of $\mathfrak{k}^{n}$, and $\tau$ induces an automorphism $\tau^{\prime}$ of $\mathfrak{k}^{m}$ which permutes the simple components cyclically. By the second duality mentioned above, there is a unique lifting $\tau_{0}: R_{0} \rightarrow R_{0}$ of $\tau^{\prime}$ along the $R$-algebra epimorphism

$$
R_{0}:=R^{m} \rightarrow \mathfrak{k}^{m} .
$$

If $\varrho \in \mathfrak{p} \backslash \mathfrak{p}^{2}$ is a uniformizing element of $R$, we define the hereditary $R$-order $\Gamma_{m}$ in $\mathrm{M}_{m}(K)$ as a crossed product

$$
\Gamma_{m}:=R_{0} \oplus R_{0} \sigma \oplus \ldots \oplus R_{0} \sigma^{m-1}
$$

with

$$
\sigma^{m}=\varrho, \quad \sigma a=\tau_{0}(a) \sigma
$$

for each $a \in R_{0}$. (If $a=\left(a_{1}, \ldots, a_{m}\right) \in R_{0}$, then (7) implies $(a \sigma)^{m}=$ $a_{1} \ldots a_{m} \cdot \varrho$. Hence $\Gamma_{m}$ does not depend on the choice of $\varrho$.) From (7) we infer

$$
\operatorname{Rad} \Gamma_{m}=\operatorname{Rad} R_{0} \oplus R_{0} \sigma \oplus \ldots \oplus R_{0} \sigma^{m-1},
$$

whence $\Gamma_{m}$ is totally split with $\Gamma_{m} / \operatorname{Rad} \Gamma_{m}=\mathfrak{k}^{m}$ according to (5). Thus for each cycle in $C$, we have constructed an $R$-order (6), and the product of these orders yields an $R$-order $\Gamma_{C}$ with $\Gamma_{C} / \operatorname{Rad} \Gamma_{C}=\mathfrak{k}^{n}$. Now the order $\Lambda_{H}$ is obtained by the pullback:

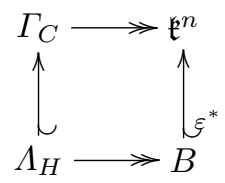

Let us reflect a bit upon this construction. By (7), the inner automorphism $a \mapsto \sigma a \sigma^{-1}$ of $\Gamma_{m}$ induces the automorphism $\tau_{0}$ on $R_{0}$, hence the automorphism $\tau^{\prime}$ on $\mathfrak{k}^{m}$. Therefore, the cycle $\mathbf{Z}_{m}$ and the hereditary order $\Gamma_{m}$ determine each other. 
There is another way to obtain $\Gamma_{m}$. Let $S$ be a $K$-vector space of dimension $m$, and let

$$
P_{0} \supset P_{1} \supset \ldots \supset P_{m}=\mathfrak{p} P_{0}
$$

be a composition series of full $R$-lattices in $S$. Define $P_{i+j m}:=\mathfrak{p}^{j} P_{i}$ for all $j \in \mathbb{Z}$. Then the $P_{i}, i \in \mathbb{Z}$, constitute an infinite chain

$$
\ldots \supset P_{-1} \supset P_{0} \supset P_{1} \supset \ldots
$$

with simple quotients $P_{i} / P_{i+1}$, and $P_{i+m}=\mathfrak{p} P_{i}$ for all $i \in \mathbb{Z}$. Clearly, if $x_{1}, \ldots, x_{m} \in S$ are chosen such that $x_{i} \in P_{i-1} \backslash P_{i}$, then they form a $K$ basis of $S$. Therefore, up to an automorphism of $S$, a chain (11) is unique. In particular, there exists a $K$-linear automorphism $\sigma$ of $S$ with

$$
\sigma P_{i}=P_{i+1}, \quad i \in \mathbb{Z} .
$$

Then the hereditary order $\Gamma_{m}$ is given by

$$
\Gamma_{m}=\left\{a \in \operatorname{End}_{K}(S) \mid a P_{i} \subset P_{i} \text { for all } i \in \mathbb{Z}\right\},
$$

and $P_{1}, \ldots, P_{m}$ represent the isomorphism classes of indecomposable $\Gamma_{m^{-}}$ representations. Thus we may assume

$$
\Gamma_{m}=P_{1} \oplus \ldots \oplus P_{m} .
$$

Now (12) implies $\sigma \Gamma_{m}=J_{m}:=\operatorname{Rad} \Gamma_{m}$, and therefore $\Gamma_{m} \sigma \subset \sigma \Gamma_{m}$. Hence $\Gamma_{m} \subset \sigma \Gamma_{m} \sigma^{-1}$ and thus

$$
\sigma \Gamma_{m}=\Gamma_{m} \sigma=J_{m}
$$

Consequently, the inner automorphism $a \mapsto \sigma a \sigma^{-1}$ of $\Gamma_{m}$ permutes the $P_{i}$ in (14) cyclically and induces an automorphism on $\Gamma_{m} / J_{m}=\mathfrak{k}^{m}$ corresponding to a cycle $\mathbf{Z}_{m}$.

As above, let $\Gamma_{C}$ be the product of these $\Gamma_{m}$, according to the cycles $\mathbf{Z}_{m}$ in $C$, and $J_{C}:=\operatorname{Rad} \Gamma_{C}$. Then (15) generalizes to

$$
\omega \Gamma_{C}=\Gamma_{C} \omega=J_{C}
$$

for some invertible element $\omega$ of $A:=K \Gamma_{C}$, and the inner automorphism $a \mapsto \omega a \omega^{-1}$ of $\Gamma_{C}$ induces the automorphism $\tau$ of $\mathfrak{k}^{n}=\Gamma_{C} / J_{C}$. Hence the permutation space $C$ is recovered from $\Gamma_{C}$.

Next let us focus our attention upon the pullback (9). Recall that an $R$ order $\Lambda$ is said to be a Bäckström order if there exists a hereditary overorder $\Gamma$ such that $\operatorname{Rad} \Gamma=\operatorname{Rad} \Lambda$.

LEMMA. For a Bäckström order $\Lambda$, the hereditary overorder $\Gamma$ with $\operatorname{Rad} \Gamma=\operatorname{Rad} \Lambda$ is unique.

Proof. For any simple $K \Lambda$-module $S$, let $\mathfrak{S}_{\Lambda}(S)$ be the set of (non-zero) $\Lambda$-lattices in $S$. The uniqueness of $\Gamma$ then follows from

$$
\mathfrak{S}_{\Gamma}(S)=\left\{I \in \mathfrak{S}_{\Lambda}(S) \mid \forall I^{\prime} \in \mathfrak{S}_{\Lambda}(S): I^{\prime} \subset I \text { or } I^{\prime} \supset I\right\}
$$


since $\Gamma$ is the multiplier of its indecomposables (cf. (13)). Now $\mathfrak{S}_{\Gamma}(S)$ is a chain, and for each $I \in \mathfrak{S}_{\Lambda}(S),(\operatorname{Rad} \Gamma) I \subset I \subset \Gamma I$. Hence the inclusion " $\subset$ " in (17) follows. Conversely, let $I$ be in the right-hand side of (17). Denote by $\Delta$ the unique maximal order in $D:=\left(\operatorname{End}_{K \Lambda} S\right)^{\mathrm{op}}$, and $\Pi:=\operatorname{Rad} \Delta$. Suppose $\alpha \in \Delta$ and $I \alpha \not \subset I$. Then $I \subset I \alpha \subset I \alpha^{2} \subset \ldots \subset I \Delta$. Hence $I=I \alpha$, and it follows that $I \Delta=I$. Since $\Gamma I /(\operatorname{Rad} \Gamma) I$ is semisimple as a $\Lambda$-module, there exists an $I^{\prime} \in \mathfrak{S}_{\Lambda}(S)$ with $I+I^{\prime}=\Gamma I$ and $I \cap I^{\prime}=(\operatorname{Rad} \Gamma) I$. By assumption, $I$ and $I^{\prime}$ are comparable, whence $I \in \mathfrak{S}_{\Gamma}(S)$.

Recall that a digraph is a graph whose vertex set is a disjoint union $X \amalg Y$ such that every edge $e$ connects a vertex $x \in X$ to a vertex $y \in Y$. If, in addition, every such $e$ is equipped with multiplicities $e x, e y \in \mathbb{N} \backslash\{0\}$, we shall speak of a di-hypergraph. Such a hypergraph can be drawn by attaching the value $(e x, e y)$ to every edge $e$ between $x$ and $y$. By convention, the value $(1,1)$ is always omitted.

With any Bäckström order $\Lambda$, a di-hypergraph $\mathrm{G}_{\Lambda}$ is naturally associated as follows (cf. [6]). If $\mathcal{Q}_{\Lambda}$ denotes the set of isomorphism classes $[U]$ of simple $\Lambda$-modules $U$, then the vertex set of $\mathrm{G}_{\Lambda}$ is the disjoint union $\mathcal{Q}_{\Lambda} \amalg \mathcal{Q}_{\Gamma}$, where $\Gamma$ is given by the lemma. By abuse we simply write $U$ instead of $[U]$. Now we define $\mathbb{Z}$-linear maps

$$
\mathbb{Z} \mathcal{Q}_{\Lambda} \stackrel{\mathrm{d}_{\Gamma}^{\Lambda}}{\longrightarrow} \mathbb{Z} \mathcal{Q}_{\Gamma} \stackrel{\mathrm{d}_{\Lambda}^{\Gamma}}{\longrightarrow} \mathbb{Z} \mathcal{Q}_{\Lambda}
$$

as follows. For $U \in \mathcal{Q}_{\Lambda}$, let $P_{U}$ be a projective cover. Then $\Gamma P_{U}$ is the projective cover of a semisimple $\Gamma$-module $\bigoplus_{V \in \mathcal{Q}_{\Gamma}} V^{\mathrm{d}_{V U}}$, and for $V \in \mathcal{Q}_{\Gamma}$, $\mathrm{d}_{U V}$ denotes the multiplicity of $U$ in a composition series of the $\Lambda$-module $V$. Then

$$
\mathrm{d}_{\Gamma}^{\Lambda}(U):=\sum_{V \in \mathcal{Q}_{\Gamma}} \mathrm{d}_{V U} \cdot V, \quad \mathrm{~d}_{\Lambda}^{\Gamma}(V):=\sum_{U \in \mathcal{Q}_{\Lambda}} \mathrm{d}_{U V} \cdot U .
$$

The maps (18) are represented in the di-hypergraph $\mathrm{G}_{\Lambda}$ by drawing an edge with value $\left(\mathrm{d}_{U V}, \mathrm{~d}_{V U}\right)$ between each pair of vertices $U, V$. Note that the maps (18) are adjoint to each other:

$$
\left\langle\mathrm{d}_{\Gamma}^{\Lambda} U, V\right\rangle_{\Gamma}=\left\langle U, \mathrm{~d}_{\Lambda}^{\Gamma} V\right\rangle_{\Lambda},
$$

where the scalar products are given by the intertwining numbers:

$$
\left\langle U, U^{\prime}\right\rangle_{\Lambda}:=\operatorname{dim}_{\mathfrak{k}} \operatorname{Hom}_{\Lambda}\left(U, U^{\prime}\right) .
$$

Equivalently, (20) says that

$$
\mathrm{d}_{V U} \cdot \operatorname{dim}_{\mathfrak{k}} \operatorname{End}_{\Gamma}(V)=\mathrm{d}_{U V} \cdot \operatorname{dim}_{\mathfrak{k}} \operatorname{End}_{\Lambda}(U)
$$

for each pair $U \in \mathcal{Q}_{\Lambda}, V \in \mathcal{Q}_{\Gamma}$. Hence $\mathrm{d}_{U V}=0$ if and only if $\mathrm{d}_{V U}=0$. Thus if for each pair $\left(\mathrm{d}_{U V}, \mathrm{~d}_{V U}\right) \neq(0,0)$, an edge $e$ with multiplicities $e U:=\mathrm{d}_{U V}$ and $e V:=\mathrm{d}_{V U}$ is introduced, we obtain a di-hypergraph $\mathrm{G}_{\Lambda}$ which is known as the valued graph of $\Lambda$ (see [6]). 
By (9), the orders $\Lambda=\Lambda_{H}$ are Bäckström orders, and we shall see that their di-hypergraph $\mathrm{G}_{\Lambda}$ has the property that all edges are of value $(1,1)$, and there is only one edge starting from any vertex of $\mathcal{Q}_{\Gamma}$ :

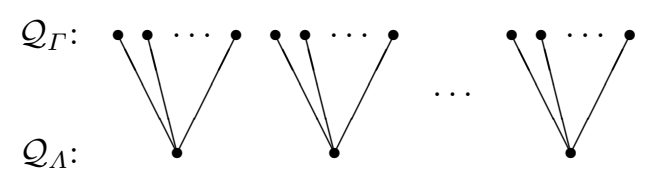

Such Bäckström orders $\Lambda$ will be called of fan type.

THEOREM 1. The correspondence $H \mapsto \Lambda_{H}$ defines a bijection between the finite cycle hypergraphs $H$ and the isomorphism classes of totally split Bäckström orders of fan type. Moreover, for an $R$-order $\Lambda$, the following are equivalent:

(a) $\Lambda$ is of the form $\Lambda_{H}$ for a finite cycle hypergraph $H$.

(b) $\Lambda$ is a Bäckström order with a totally split hereditary order $\Gamma$.

(c) $\Lambda$ is a totally split Bäckström order of fan type.

Proof. The implication (a) $\Rightarrow$ (b) follows by the construction of $\Lambda_{H}$.

(b) $\Leftrightarrow(\mathrm{c})$. If $\Gamma$ is totally split, then each simple $\Gamma$-module $V$ coincides with a simple $\Lambda$-module $U$. Hence there is exactly one edge in $\mathrm{G}_{\Lambda}$ starting from $V$, and $\mathrm{d}_{U V}=1$. Since $V=\mathfrak{k}$, we also infer $\mathrm{d}_{V U}=1$, and $\Lambda$ is totally split. The converse follows immediately by $(22)$.

(b) $\Rightarrow(\mathrm{a})$. We have already shown that there is a bijective correspondence between totally split hereditary orders $\Gamma$ and finite permutation spaces $C$. The Bäckström orders for such a $\Gamma$ correspond to the (necessarily semisimple) $\mathfrak{k}$-subalgebras $B$ of $\Gamma / \operatorname{Rad} \Gamma=\mathfrak{k}^{n}$, and $\Lambda$ is given by a pullback (9). This also shows that $\Lambda$ uniquely determines the cycle hypergraph $H$ with $\Lambda \cong \Lambda_{H}$.

As a consequence we note that the hereditary order $\Gamma$ belonging to $\Lambda_{H}$ is a hereditary hull [3] of $\Lambda$, i.e. $\Gamma$ is a minimal hereditary overorder of $\Lambda$ :

Corollary. If $\Lambda_{H}$ is the order of a cycle hypergraph $H$ given by $\varepsilon$ : $C \rightarrow E$, then $\Gamma_{C}$ is the unique hereditary hull of $\Lambda_{H}$.

P r o of. The property of $\Gamma$ to be a hereditary hull of an $R$-order $\Lambda$ means that for each simple $K \Gamma$-module $S$, the chain $\mathfrak{S}_{\Gamma}(S)$ cannot be refined by some $I \in \mathfrak{S}_{\Lambda}(S)$ with $I \Delta=I$, where $\Delta$ denotes the maximal order in $D:=\left(\operatorname{End}_{K \Gamma} S\right)^{\mathrm{op}}$. This is obviously satisfied for a totally split hereditary overorder $\Gamma$.

3. Prohereditary rings. Having associated an order $\Lambda_{H}$ with any finite cycle hypergraph $H$, our next purpose, going in reverse direction, will be to introduce a general class of rings for which an underlying cycle hypergraph $H$ can be naturally defined. To this end, we start with a generalization 
of hereditary orders and QH-orders [8]. Let $S$ be a semiperfect ring. For a finitely generated projective $S$-module $P$, let us call a homomorphism $P \rightarrow M$ into an $S$-module $M$ indirect if it factors through an indecomposable projective $S$-module $Q$ which is not a direct summand of $P$. For a given $M$, the sum of all images of indirect homomorphisms $P \rightarrow M$ will be called the proradical Pro $M$ of $M$. Clearly, this definition is functorial, i.e. for any homomorphism $f: M \rightarrow N$ of $S$-modules, $f(\operatorname{Pro} M) \subset$ Pro $N$. In particular, this implies that Pro $S:=\operatorname{Pro}\left({ }_{S} S\right)$ is a (two-sided) ideal of $S$. Moreover, we have

Proposition 2. For every semiperfect ring $S$, the proradical is contained in the Jacobson radical $\operatorname{Rad} S$, and $S / P r o S$ is a product of matrix rings over local rings.

Proof. Since each indirect homomorphism $P \rightarrow Q$, with $Q$ indecomposable and projective, maps into the radical of $Q$, we have $\operatorname{Pro} S \subset \operatorname{Rad} S$. Secondly, each indirect homomorphism $P \rightarrow S / \operatorname{Pro} S$ is zero since it factors through the natural epimorphism $S \rightarrow S /$ Pro $S$. Hence

$$
\operatorname{Pro}(S / \operatorname{Pro} S)=0 \text {. }
$$

Therefore, it remains to prove that $S$ is a product of matrix rings over local rings whenever Pro $S=0$. Now this condition says that there are no nonzero homomorphisms between any pair of non-isomorphic indecomposable projective $S$-modules. Hence, $S=\left(\operatorname{End}_{S} S\right)^{\text {op }}$ gives the desired result.

Let us call an ideal $I$ of $S$ invertible if ${ }_{S} I$ is a progenerator (i.e. a finitely generated projective generator of $S$-Mod) with $\left(\operatorname{End}_{S} I\right)^{\text {op }}=S$ (i.e. the restriction $S=\left(\operatorname{End}_{S} S\right)^{\text {op }} \rightarrow\left(\operatorname{End}_{S} I\right)^{\text {op }}$ is a ring isomorphism). Then we define a prohereditary ring as a semiperfect ring $S$ with an invertible ideal $J$ such that Pro $S \subset J \subset \operatorname{Rad} S$ and

$$
\bigcap_{i=0}^{\infty} J^{i}=0 .
$$

We refer to $J$ as the defining ideal of $S$. If $J$ coincides with the proradical, then $S$ will be called strictly prohereditary. Thus a local ring $S$ is prohereditary if and only if it has an invertible ideal $J$ which satisfies (24). Note that in this case, Pro $S=0 \neq J$.

Proposition 3 (cf. [7], Theorem 1.5). For a basic semiperfect ring $S$, the following are equivalent:

(a) $S$ is prohereditary.

(b) There is a left projective ideal $J \subset \operatorname{Rad} S$ satisfying (24) such that $\left(\operatorname{End}_{S} J\right)^{\mathrm{op}}=S$, and $S / J$ is a product of local rings. 
(c) $S$ is a product of rings of the form

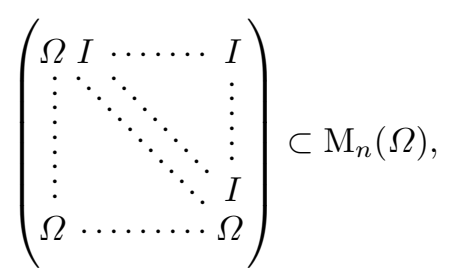

where $n \geq 1$, and $\Omega$ is local prohereditary with defining ideal I.

Note. The proposition shows that QH-orders [7, 8] over a complete noetherian local domain $R$ are prohereditary.

Proof. $(\mathrm{a}) \Rightarrow(\mathrm{b})$ (of Proposition 3 ). This follows immediately by Proposition 2 .

(b) $\Rightarrow(\mathrm{c})$. For each indecomposable projective $S$-module $P$, the submodule $J P=J \otimes_{S} P$ is projective, and $\operatorname{Hom}_{S}\left(J, J \otimes_{S} P\right)=\operatorname{Hom}_{S}(J, J) \otimes_{S} P=$ $S \otimes_{S} P=P$. Hence, $J P$ is again indecomposable, and $P \mapsto J P$ yields a permutation on the isomorphism classes of indecomposable projective $S$ modules. Therefore, we get a set of pairwise disjoint cycles $P \supsetneqq J P \supsetneqq J^{2} P \supsetneqq$ $\ldots \supsetneqq J^{n} P \cong P$ of indecomposable projectives. If $Q$ belongs to a different cycle, then each homomorphism $Q \rightarrow P$ induces a map $Q / J Q \rightarrow P / J P$ which must be zero by the assumption that $S / J$ is a product of local rings. Hence, $Q$ maps into $J P$, and by induction, the image of $Q$ lies in $\bigcap J^{i} P=0$. Therefore, let us assume that there is just one cycle, i.e. ${ }_{S} S \cong P \oplus J P \oplus \ldots \oplus J^{n-1} P$. By the above, we have $\operatorname{Hom}_{S}\left(J \otimes_{S} P, J P\right)=\operatorname{Hom}_{S}\left(P, \operatorname{Hom}_{S}(J, J P)\right)=$ $\operatorname{Hom}_{S}(P, P)$, whence there is a common endomorphism ring

$$
\Omega:=\operatorname{End}_{S}(P)=\operatorname{End}_{S}(J P)=\ldots=\operatorname{End}_{S}\left(J^{n-1} P\right) .
$$

For $i, j \in\{0, \ldots, n-1\}$ with $i>j$, we have

and

$$
\operatorname{Hom}_{S}\left(J^{i} P, J^{j} P\right)=\operatorname{Hom}_{S}\left(J^{i} P, J^{i} P\right)=\Omega,
$$

$$
\operatorname{Hom}_{S}\left(J^{j} P, J^{i} P\right)=\operatorname{Hom}_{S}\left(J^{j} P, J^{j+n} P\right)=\operatorname{Hom}_{S}\left(P, J^{n} P\right)=: I,
$$

where $I \cong \Omega$ is invertible with $\bigcap I^{i}=0$.

(c) $\Rightarrow($ a). We may assume that $S$ is of the matrix form given in (c), and $n \geq 2$. Then ${ }_{\Omega} I \cong{ }_{\Omega} \Omega$ since $\Omega$ is local, and

$$
\text { Pro } S=\left(\begin{array}{ccccc}
I & \cdots & \cdots & \cdots & I \\
\Omega & \ddots & & & \vdots \\
\vdots & \ddots & \ddots & & \vdots \\
\vdots & & \ddots & \ddots & \vdots \\
\Omega & \cdots & \cdots & \Omega & I
\end{array}\right) \cong S
$$

as a left $S$-module, whence $J:=\operatorname{Pro} S$ is invertible and satisfies (24). 
As an immediate consequence, we note:

COROLlary. Let $S$ be a semiperfect ring. Then

(a) If $S$ is prohereditary, then every ring $S^{\prime}$ Morita equivalent to $S$ is prohereditary.

(b) If $S$ is basic and prohereditary, then $S$ is strictly prohereditary if and only if $S$ has no local ring-direct factors.

(c) If $S$ is basic and prohereditary with defining ideal $J$, then $J=S \omega=$ $\omega S$ for some regular element $\omega \in \operatorname{Rad} S$.

Proof. (a) and (b) follow by the preceding proof. For the proof of (c), let $S$ be basic and prohereditary. Then ${ }_{S} J \cong{ }_{S} S$ by Proposition 3(c), i.e. $J=S \omega$, where the right multiplication by $\omega$ is injective. Since $\left(\operatorname{End}_{S} J\right)^{\mathrm{op}}=$ $S$, we infer that $S \omega=\omega S$, and $\omega$ is regular.

4. Rings with Green walks. Let $\Gamma$ be a prohereditary ring with defining ideal $J$. The $\Gamma$-modules of the form $Q / J Q$ with $Q$ indecomposable projective will be called prosimple. Let $\mathcal{Q}_{\Gamma}$ be a representative system of the isomorphism classes of prosimple $\Gamma$-modules. Generalizing the concept of Bäckström order, we call a semiperfect subring $\Lambda$ of $\Gamma$ a Bäckström ring with respect to $\Gamma$ if Pro $\Lambda \subset J \subset \Lambda$, and $\Gamma / J$ is a finite direct sum of $\Lambda$ modules isomorphic to some $P / J P$ with ${ }_{\Lambda} P$ indecomposable and projective.

Let $\mathcal{Q}_{\Lambda}$ be a representative system for the isomorphism classes of these $\Lambda$ modules $P / J P$. Just as in $\S 2$, we define a di-hypergraph $G_{\Lambda}^{\Gamma}$ with vertex set $\mathcal{Q}_{\Lambda} \amalg \mathcal{Q}_{\Gamma}$, and for $(U, V) \in \mathcal{Q}_{\Lambda} \times \mathcal{Q}_{\Gamma}$, we introduce an edge with multiplicity $\left(\mathrm{d}_{U V}, \mathrm{~d}_{V U}\right)$, where $\mathrm{d}_{U V}$ is the multiplicity of $U$ in ${ }_{\Lambda} V$, and if $P_{U}, P_{V}$ are projective covers of $U$ and $V$, respectively, then $\mathrm{d}_{V U}$ denotes the multiplicity of $P_{V}$ in $\Gamma P_{U}$.

Motivated by Theorem 1, we now define a ring with Green walks as a Bäckström ring $\Lambda$ with respect to a prohereditary ring $\Gamma$ such that $G_{\Lambda}^{\Gamma}$ is of the form (23). Clearly, there is no loss of generality if we assume $\Lambda$ to be basic. Then the latter condition says that $\Gamma$ is also basic, and that for each $V \in \mathcal{Q}_{\Gamma}$, the $\Lambda$-module ${ }_{\Lambda} V$ lies in $\mathcal{Q}_{\Lambda}$.

Note. In contrast to Bäckström orders, a ring $\Lambda$ with Green walks does not always determine $\Gamma$. Therefore, we have to consider $\Lambda$ in conjunction with its prohereditary overring $\Gamma$, and the defining ideal $J$ of $\Gamma$. These data will be kept fixed throughout the sequel.

Next we define the cycle hypergraph $H_{\Lambda}^{\Gamma}$ of $\Lambda$ (with respect to $\Gamma$ and $J$ ). Firstly, the map $Q \mapsto J Q$ for indecomposable projective $\Gamma$-modules $Q$ induces a permutation $\pi$ on $\mathcal{Q}_{\Gamma}$ and turns $C:=\mathcal{Q}_{\Gamma}$ into a permutation space. Moreover, with $E:=\mathcal{Q}_{\Lambda}$, the di-hypergraph (23) is equivalent to a map

$$
\varepsilon: C \rightarrow E,
$$


and thus we obtain a finite cycle hypergraph $H=H_{\Lambda}^{\Gamma}$. The edges $e \in E$ of $H$ correspond to the simple $\Lambda$-modules or equivalently, to the $\Lambda$-modules $U_{e}:=P_{e} / J P_{e} \in \mathcal{Q}_{\Lambda}$ and their projective covers $P_{e}$, the elements $c \in C$ correspond to the indecomposable projective $\Gamma$-modules $P_{c}$, and the vertices $x \in X$, i.e. the cycles of $C$, correspond to the indecomposable ring-direct summands of $\Gamma$. Hence

$$
J P_{c}=P_{\pi c},
$$

and the projective resolutions of the $U_{e}$ are given by the short exact sequences

$$
\begin{aligned}
& \bigoplus_{\varepsilon(d)=e} P_{\pi d} \longmapsto P_{e} \rightarrow U_{e} \\
& \bigoplus_{\substack{\varepsilon(d)=e \\
d \neq c}} P_{\pi d} \longmapsto P_{e} \rightarrow P_{c}
\end{aligned}
$$

for each $e \in E$ and $c \in \varepsilon^{-1}(e)$. To obtain (27), consider a homomorphism $f$ : $P_{e} \rightarrow P_{c}$ of $\Lambda$-modules which induces an isomorphism $P_{e} / J P_{e} \stackrel{\sim}{\longrightarrow} P_{c} / J P_{c}$. Then $f$ extends to a homomorphism $\Gamma P_{e} \rightarrow P_{c}$ of $\Gamma$-modules which must be surjective by Nakayama's lemma. Therefore, $J P_{e}$ is mapped onto $J P_{c}$, whence $f$ is surjective, and the exactness of (27) follows.

By these exact sequences, the rôle of the resolution graph $W_{H}$ defined in $\S 1$ becomes apparent. Namely, its arrows just give the syzygies according to (27). Together with $H$, the resolution graph $W_{H}$ yields the projectives as well as the syzygies of a projective resolution of $\Lambda / J$. Moreover, the arrows in $W_{H}$ also give some information about the maps in this projective resolution. Therefore, $W_{H}$ exactly describes what could be said to be the combinatorial structure of a projective resolution of $\Lambda / J$. In particular, the preceding discussion yields:

THEOREM 2. For a ring $\Lambda$ with Green walks and underlying cycle hypergraph $H$, the global dimension of $\Lambda$ is infinite if the resolution graph $W_{H}$ contains an oriented cycle; otherwise, gld $\Lambda$ is the maximum number of vertices in a directed path of $W_{H}$. For each vertex $c$ of $W_{H}$, there are $\mathrm{r}(\varepsilon(c))-1$ arrows starting from $c$, and $\mathrm{r}\left(\varepsilon\left(\pi^{-1} c\right)\right)-1$ arrows ending in $c$. There is no loop in $W_{H}$ if and only if $\varepsilon(\pi c) \neq \varepsilon(c)$ for each $c \in C$.

Hence if the rank of $H$ is $\leq 2$, every path in $W_{H}$ has at most one continuation on and backwards, which implies Roggenkamp's result [8]:

COROLlary. If $H$ is of rank $\leq 2$, then $W_{H}$ is a disjoint union of oriented cycles and finite paths. Accordingly, the corresponding projective resolutions are either cyclic or bounded of the form

$$
0 \rightarrow P_{e_{m}} \rightarrow \ldots \rightarrow P_{e_{0}} \rightarrow U_{e_{0}}
$$


with $e_{0}, \ldots, e_{m} \in E$ and 1-loops $e_{0}$ and $e_{m}$. Moreover, in the totality of these projective resolutions, every edge $e \in E$ occurs exactly twice.

Note. By the results of $\S 2$, each cycle hypergraph $H$ arises from a ring with Green walks, for instance from the totally split $R$-order $\Lambda_{H}$ for any complete discrete valuation domain $R$.

EXAMPLE 2. For the cycle hypergraph $H$ of Example 1, the resolution graph $W_{H}$ consists of two chains:

$$
5 \rightarrow 1 \rightarrow 6 \rightarrow 4, \quad 3 \rightarrow 2 .
$$

Hence gld $\Lambda_{H}=4$, and similarly for each ring with Green walks and underlying cycle hypergraph $H$. Applying $\varepsilon$ we get the projective resolutions

$$
P_{f} \longmapsto P_{h} \rightarrow P_{e} \rightarrow P_{e} \rightarrow P_{5}, \quad P_{g} \longmapsto P_{h} \rightarrow P_{3},
$$

which can be completed by (26) to

$$
0 \rightarrow P_{f} \rightarrow P_{h} \rightarrow P_{e} \rightarrow P_{e} \rightarrow P_{f} \rightarrow U_{f}, \quad 0 \rightarrow P_{g} \rightarrow P_{h} \rightarrow P_{g} \rightarrow U_{g} .
$$

Here every edge of $H$ occurs exactly twice.

EXAMPLE 3 . Consider the following cycle hypergraphs $H_{1}, H_{2}, H_{3}$ :
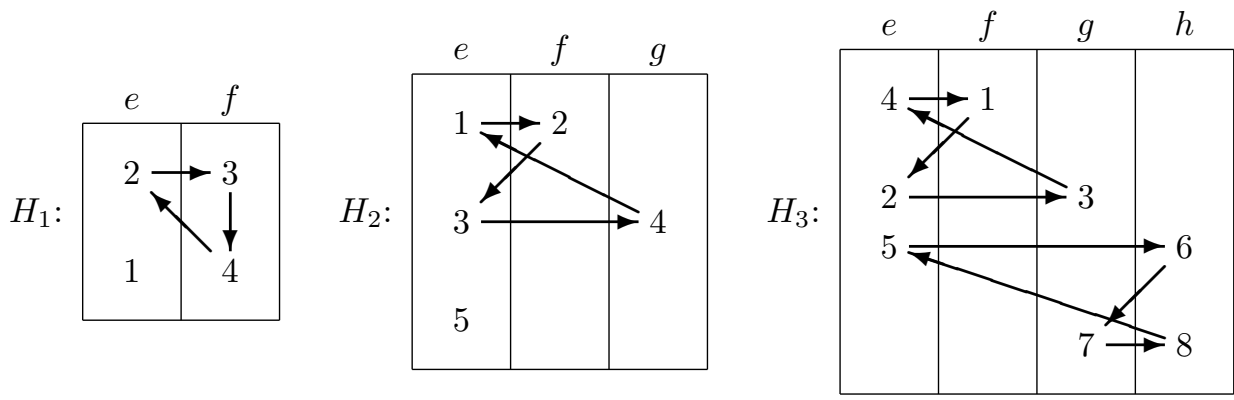

$H_{1}$ corresponds to the totally split $R$-order:

$$
\Lambda_{H_{1}}=\left(\begin{array}{ccc}
R & \mathfrak{p} & \mathfrak{p} \\
R & R & \mathfrak{p} \\
R & R & R
\end{array}\right), \quad R-R:=\{(a, b) \in R \times R \mid a-b \in \mathfrak{p}\} .
$$

The resolution graphs of $H_{1}, H_{2}, H_{3}$ are

$W_{H_{1}}$ :

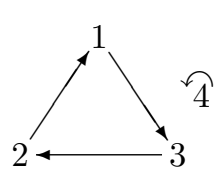

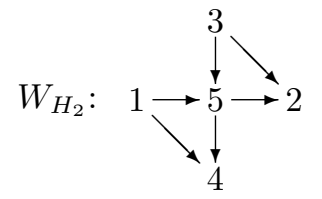

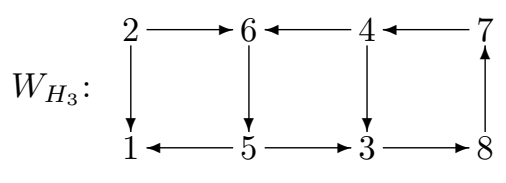

$W_{H_{1}}$ has a loop since $\varepsilon^{-1}(f)$ contains the arrow $3 \rightarrow 4 ; \Lambda_{H_{2}}$ is of global dimension three, whereas gld $\Lambda_{H_{3}}=\infty$ since $W_{H_{3}}$ contains an oriented cycle. 
Note that for a cycle hypergraph $H$ of arbitrary rank, the order $\Lambda_{H}$ may have infinitely many indecomposable representations. Nevertheless, the global dimension of $\Lambda_{H}$ does not depend on $R$, in contrast e.g. to tiled orders [9]. For an arbitrary Bäckström order $\Lambda$ with hereditary order $\Gamma$, Wiedemann [11] has shown that whenever gld $\Lambda$ is finite, it is bounded by the number of indecomposable $\Gamma$-lattices. For $\Lambda_{H}$ this follows immediately by Proposition 2:

$$
\operatorname{gld} \Lambda_{H} \leq|C|
$$

It is easy to see that the maximum is attained for every given permutation space $C$. The simplest way to achieve this is to build a tree with the given vertices by means of edges of rank two and to complete it by adding a single 1-loop. (There are other possibilities to get equality in (28), but the rank of $H$ has to be two for this purpose!)

\section{REFERENCES}

[1] C. Berge, Graphes et hypergraphes, Dunod, Paris, 1973.

[2] J. A. Green, Walking around the Brauer tree, J. Austral. Math. Soc. 17 (1974), 197-213.

[3] H. Jacobinski, Maximalordnungen und erbliche Ordnungen, Vorl. FB-Math. Univ. Essen, Heft 6, 1981.

[4] W. Plesken, Group Rings of Finite Groups over p-adic Integers, Lecture Notes in Math. 1026, Springer, Berlin, 1983.

[5] I. Reiner, Maximal Orders, Academic Press, London, 1975.

[6] C. M. Ringel and K. W. Roggenkamp, Diagrammatic methods in the representation theory of orders, J. Algebra 60 (1979), 11-42.

[7] K. W. Roggenkamp, Blocks of cyclic defect and Green-orders, Comm. Algebra 20 (1992), 1715-1734.

[8] —, Generalized Brauer tree orders, Colloq. Math. 71 (1996), 225-242.

[9] W. Rump, Discrete posets, cell complexes, and the global dimension of tiled orders, Comm. Algebra 24 (1996), 55-107.

[10] D. J. A. Welsh, Matroid Theory, Academic Press, London, 1976.

[11] A. Wiedemann, Projective resolutions and the global dimension of subhereditary orders, Arch. Math. (Basel) 53 (1989), 461-468.

Mathematisch-Geographische Fakultät

Katholische Universität Eichstätt

Ostenstraße 26-28

D-85071 Eichstätt, Germany

E-mail: wolfgang.rump@ku-eichstaett.de 